\title{
Characterization of suspicious breast lesions with dynamic contrast enhanced MRI in comparison to conventional mammography and ultrasonography
}

\begin{abstract}
This study included thirty three patients with suspicious breast lesions. For every patient; Mammography, Ultrasonography and Dynamic MRI were done.Our results indicate that the sensitivity of breast imaging can be increased by complementary use of MRI. For patients in whom the status of breast lesion remains unclear, MRI, though most expensive among all other complementary diagnostic modalities, it reduces the number of unnecessary biopsies and diagnostic challenges.
\end{abstract}

Keywords: mammography, breast ultrasound, dynamic MRI breast
Volume 4 Issue 3 - 2016

\author{
Safieeldin Ghazala,' Mervat Elgohary, ${ }^{2}$ Dalia \\ Zidan, ${ }^{2}$ Mary Yaftah ${ }^{2}$ \\ 'Almostaqbal Hospital, Saudi Arabia \\ ${ }^{2}$ Department of Radiodiagnosis, Ain shams University, Egypt
}

Correspondence: Safieeldin Ghazala,Almostaqbal Hospital, Jeddah 2144I, Saudi Arabia, Email ser_128@hotmail.com

Received: December 17, 2015 | Published: February 24, 2016

\section{Introduction}

Currently, conventional mammography and sonography are the techniques most widely used for the early detection and localization of breast abnormalities. ${ }^{1}$

Mammography has long been used for early detection of and screening for breast cancers. It is believed that mammography alones misses between $10 \%$ and $30 \%$ of all breast cancers. Possible reasons may include density of breast parenchyma and slow growing breast cancers. $^{2}$

Ultrasound has been used as an adjunct to mammography, with particular value in differentiating cystic from solid lesions and in facilitating guided biopsy of suspicious areas. ${ }^{2}$

Magnetic resonance imaging (MRI) is a non invasive imaging technique that has been rapidly developed over the past decade and is regarded as the most potential examination modality for diagnosis of breast cancer. ${ }^{3}$

MRI permits us to explore two concepts: First, we are able to analyze the morphologic characteristics of the lesions with high spatial resolution, such as the margin morphology or the internal architecture of the tumors. Second, we can obtain dynamic data derived from the kinetic patterns of lesion enhancement after the administration of contrast material. ${ }^{4}$ Integration of both kinetic and morphological features is important for accurate diagnosis. ${ }^{5}$

Dynamic contrast enhanced magnetic resonance imaging (DCEMRI) is an emerging imaging method to enable the depiction of physiologic alterations and to assess tumor angiogenesis. ${ }^{6}$ This angiogenesis is often too small to be proved by another imaging method. ${ }^{7}$

Among the limitations of breast MRI are its higher cost, longer examination time, and lower availability compared with mammography and ultrasound. ${ }^{8}$

\section{Patients and methods}

The current study was performed in 33 patients with suspicious breast lesions; their mean age was 44years (age range, 35-53years). Thirty six breast lesions were detected.

The study was conducted in Almostaqbal Hospital during the period from 2011 to 2015. Cases were referred from the surgical clinics at each hospital. The patients underwent full history taking and clinical examination, X-ray mammography, US and MRI examination.

\section{Inclusion criteria}

I. Any patient who is scheduled for dynamic breast MRI to differentiate benign and malignant lesions, to preoperatively assess local extent, bilaterality and multicentricity of the tumor in patients with malignant lesions and to differentiate between post operative surgical scars from recurrent tumors.

II. No age predilection.

\section{Exclusion criteria}

I. Pregnant patients.

II. Patients with elevated renal function tests.

III. Patients who have contraindications to enter magnetic field like those with pacemaker, cochlear implant or any other metallic prosthesis that interferes with the examination.

\section{Methods}

Mammography was conducted for all patients using digital mammography in both medio lateral oblique and craniocaudal views with spot compression magnification view when necessary.

Mammographic images were analyzed regarding the presence of masses, architectural distortion, asymmetrical density and calcification. In detected masses their side, site, number, margins and density were evaluated, while in case of micro calcification their shape and distribution were assessed.

Then classification of breast lesions was based on the Breast Imaging Reporting and Data System (BI-RADS). Then ultrasound was performed for all patients using a high frequency probe (7.5MHZ), scanning was done in two orthogonal (longitudinal and transverse) planes. 
Masses were evaluated by US considering BI-RADS US lexicon regarding: their shape, orientation, margins, echopattern (anechoic, hyperechoic, complex, hypoechoic, isoechoic), lesion boundary (abrupt interface, echogenic halo) and presence or absence of acoustic shadowing or enhancement.

Then all patients were referred for further MRI examination.

\section{MR imaging protocol}

Dynamic MR imaging was performed with high field strength 1.5 Tesla on (Philips Ingenia system) using dedicated double breast coil.

Coronal T1 weighted spin echo sequence was carried out for localization purpose and followed by plain sequences using T1weighted fast spin echo sequence $(\mathrm{TR}=125 \mathrm{msec}$. $\mathrm{TE}=5.3 \mathrm{msec}$. $)$, in addition to $\mathrm{T} 2$ weighted fast spin echo sequence (TR=3740mesc., TE=90 msec.) in axial orientation.

A bolus of gadolinium dimeglumine (Gd-DTPA) (Magnavist, Schering AG Berlin, Germany) was injected manually intravenous at a dose of $(0.1 \mathrm{mmol} / \mathrm{kg})$ followed by saline flush to ensure that contrast -enhanced images could be obtained immediately after contrast agent injection.

Dynamic T1 WIs then performed using Gradient echo T1 weighted image with fat suppression at the following time points: 1 minute, 2 min., $4 \mathrm{~min}$. and $7 \mathrm{~min}$.

Post processing image subtraction was obtained between the post contrast imaging showing maximum enhancement and pre-contrast images (in the same axial plane), using the software subtraction function available on the work station.

Quantitative analysis was done by placing the region of interest (ROI) at the most enhanced part within the lesion result in automatically created time/ signal intensity curve.

\section{MRI image interpretation}

Conventional $\mathrm{T} 1$ and $\mathrm{T} 2$ weighted images were first examined to detect the presence or absence of benign lesions (e.g. cysts and fat containing lesions) then $\mathrm{T} 1$ dynamic and subtraction images were examined to detect the presence or absence of lesion enhancement.

In case of lesion enhancement the corresponding non subtracted pre-contrast and post contrast images in each time point was viewed together and lesions interpretation took place whether it is a focus, mass or non mass like enhancement.

In case of mass enhancement evaluation was carried out as follow:

I. Its shape (regular or irregular).

II. Its border (well defined, ill defined, speculated).

III. Pattern of enhancement (homogenous, heterogeneous or ring enhancement).

IV. Dynamic behavior of the mass with evaluation of the percentage of enhancement as well as the shape of time/signal intensity curve (type I, type II or type III) was studied.

V. In case of non mass like enhancement, its distribution and enhancement pattern were evaluated.

VI. MRI findings were correlated with histopathological result.

\section{Data analysis}

Data were statistically described in terms of range, mean \pm standard deviation $( \pm \mathrm{SD})$, median, frequencies (number of cases) and percentages when appropriate. Comparison between categorical data was performed using Chi square test. Accuracy was represented using the terms sensitivity, specificity, positive predictive value, negative predictive value, and overall accuracy.

All statistical calculations were done using computer programs Microsoft Excel 2007 (Microsoft Corporation, NY, USA) and SPSS (Statistical Package for the Social Science; SPSS Inc., Chicago, IL, USA) version 15 for Microsoft Windows. P value less or equal to 0.05 was considered significant and less than 0.01 was considered highly significant.

\section{Results}

The present study included 33 patients, their age ranged between 35 and 53 years (mean age 44). Thirty six breast lesions were detected.

The breast lesions were classified according to different imaging modalities using mammographic, ultrasonographic and MRI Breast Imaging Reporting and Data System.

Histopathological analysis was done for each lesion with 7 lesions $(19.4 \%)$ proved to be benign, 1 lesion $(2.4 \%)$ proved to be precancerous and 28 lesions $(77.7 \%)$ proved to be malignant, the results were correlated with the findings in each diagnostic modality. The different pathologies encountered in our study are illustrated in (Table 1 \& Figure1).

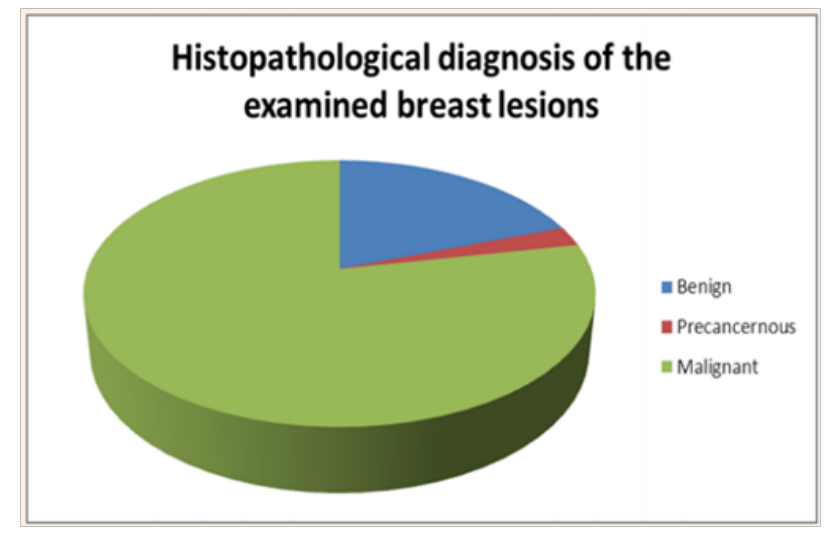

Figure I Pie chart illustrating the histopathological diagnosis of the examined breast lesions.

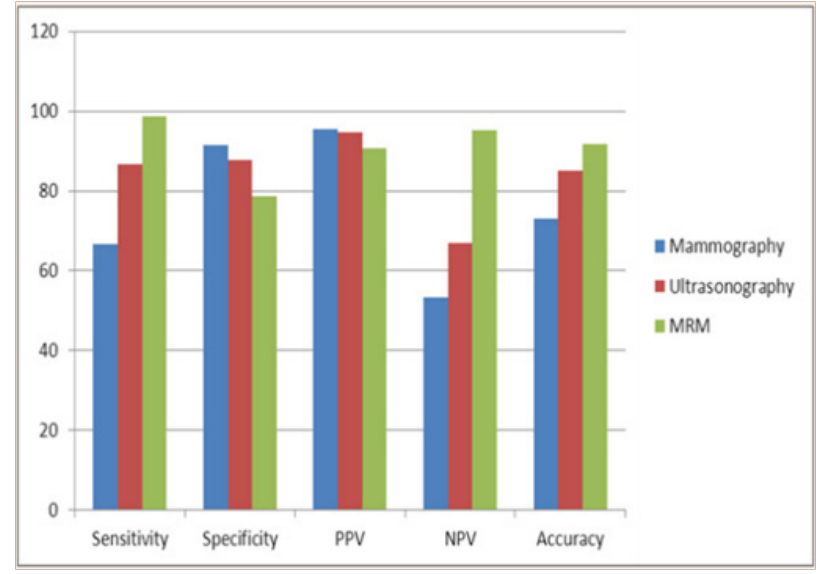

Figure 2 Clustered column chart comparing the sensitivity, specificity, PPV, NPV and accuracy of mammography, US and MRM based on BI-RADS category. 


\section{Mammographic findings}

Diagnostic mammography was the first examination performed for all patients for evaluation of their palpable breast lesions. The lesions were classified according to Breast Imaging Reporting and Data System (Table 2).

Table I Shows histopathological diagnosis of the 36 examined breast lesions

\begin{tabular}{ll}
\hline Histopathological diagnosis & Number \\
\hline Benign & $\mathbf{7}$ ( I 9.4\%) \\
Fibroadenoma & 5 \\
Diabetic Mastopathy & $\mathrm{I}$ \\
Post Operative Scar & $\mathrm{I}$ \\
Precancerous & $\mathrm{I}(\mathbf{2 . 4 \% )}$ \\
Atypical Ductal Hyperplasia & $\mathrm{I}$ \\
Malignant & $\mathbf{2 8}(\mathbf{7 7 . 7 \% )}$ \\
Invasive Ductal Carcinoma & 20 \\
Invasive lobular Carcinoma & 2 \\
Insitu Ductal Carcinoma & 4 \\
Medullary Carcinoma & $\mathrm{I}$ \\
Tubular Carcinoma & $\mathrm{I}$ \\
Total & $\mathbf{3 6}(\mathbf{~ 0 0 \% )}$
\end{tabular}

Table 2 Mammographic BI-RADS classification of the 36 examined breast lesions in relation to their histopathological results

\begin{tabular}{llllllll}
\hline Histopathology & \multicolumn{7}{c}{ Mammography } \\
& $\mathbf{0}$ & $\mathbf{I}$ & $\mathbf{2}$ & $\mathbf{3}$ & $\mathbf{4}$ & $\mathbf{5}$ & Total \\
Benign & 0 & 0 & 0 & 5 & 2 & 0 & 7 \\
Malignant & 6 & 0 & 0 & 1 & 19 & 3 & 29 \\
Total & 6 & 0 & 0 & 6 & 21 & 3 & 36 \\
& $16.7 \%$ & $0 \%$ & $0 \%$ & $16.7 \%$ & $58.3 \%$ & $8.3 \%$ & $100 \%$ \\
\hline
\end{tabular}

Mammography was inconclusive (BI-RADS 0) in 6 lesions with very dense breasts, which were malignant by histopathology.

Six lesions were classified as probably benign (BI-RADS 3), 5 of them (83.3\%) proved to be benign (true negative) and 1 lesion (16.7\%) was malignant (false negative result). BI-RADS 4 were encountered in 21 lesions $(58.3 \%), 19$ of them (90.4\%) proved to be malignant and 2 lesions (9.6\%) were benign. BI-RADS 5 was encountered in 3 lesions proved all to be malignant (true positive results).

\section{Ultrasound findings}

Ultrasound was done for all the patients with classification of the breast lesions based on US BI-RADS lexicon (Table 3).

Table 3 Shows the BI-RADS classification of breast lesions by US in relation to their histopathological results

\begin{tabular}{llllllll}
\hline Histopathology & \multicolumn{1}{l}{ US BI-RADS } \\
\hline \multirow{3}{*}{ Benign } & $\mathbf{0}$ & $\mathbf{I}$ & $\mathbf{2}$ & $\mathbf{3}$ & $\mathbf{4}$ & $\mathbf{5}$ & Total \\
Malignant & 0 & 0 & 2 & 3 & 2 & 0 & 7 \\
& 0 & 0 & 0 & 0 & 25 & 4 & 29 \\
Total & 0 & 0 & 2 & 3 & 27 & 4 & 36 \\
& $0 \%$ & $0 \%$ & $5.5 \%$ & $8.3 \%$ & $75 \%$ & $\mathbf{I} 1.2 \%$ & $100 \%$
\end{tabular}

Two lesions were classified as BI-RADS 2 lesions which were classified as BI-RADS 3 lesions by mammography and proved histologically to be benign. Four lesions were classified as BI-RADS 5 , three of them were classified as BI-RADS 4 by mammography and the fourth lesion was classified as BI-RADS 0 mamographically due to dense parenchyma, all the four lesions proved histologically to be malignant. BI-RADS 4 lesions represent 27 lesions, 2 of them (7.4
$\%$ ) were benign (false positive result) and 25 lesions (92.6\%) were malignant (true positive). Three lesions were classified as BI-RADS 3 proved to be histologically benign.

\section{Dynamic MRI findings}

All patients were referred for MRI examination and classification based on MRI BI-RADS lexicon (Table 4). Six lesions (16.6\%) were assigned as benign findings, all of them proved to be histologically benign. One lesion $(2.8 \%)$ was classified as (BI-RADS 4), which proved histologially to be benign. Three lesions were diagnosed as BI-RADS 4 (8.4\%), all of them diagnosed histopathologically as malignant. BI-RADS 5 was assigned for malignant findings for 26 lesions $(72.2 \%)$ which all proved to be malignant.

Table 4 Shows BI-RADS MR classification of breast lesions and their histopathological results

\begin{tabular}{llllllll}
\hline Histopathology & \multicolumn{1}{l}{ MRI BI-RADS } \\
\hline \multirow{3}{*}{ Benign } & $\mathbf{0}$ & $\mathbf{I}$ & $\mathbf{2}$ & $\mathbf{3}$ & $\mathbf{4}$ & $\mathbf{5}$ & Total \\
Malignant & 0 & 0 & 6 & 0 & $\mathrm{I}$ & 0 & 7 \\
Total & 0 & 0 & 0 & 0 & 3 & 26 & 29 \\
& 0 & 0 & 6 & 0 & 4 & 26 & 36 \\
& $0 \%$ & $0 \%$ & $16.6 \%$ & $0 \%$ & $11.2 \%$ & $72.2 \%$ & $100 \%$
\end{tabular}

Breast lesions were interpreted by MRI examination as focus (2 lesions), mass (28 lesions) or non mass like enhancement (6 lesions) (Table 5). Five lesions were interpreted as non mass like enhancement, all were histologically malignant, 2 of them had linear ductal enhancement and 3 had segmental stippled enhancement.

Table 5 Shows MRI interpretation of breast lesions in correlation with histopathology

\begin{tabular}{|c|c|c|c|}
\hline \multirow{2}{*}{ MRI interpretation } & \multicolumn{3}{|c|}{ Histopathology } \\
\hline & Benign & Malignant & Total \\
\hline Focus & 0 & 2 & $2(5.5 \%)$ \\
\hline Mass & 7 & 22 & 29 (80.5\%) \\
\hline Non mass & 0 & 5 & $5(14.0 \%)$ \\
\hline Total & 7 & 29 & 36 \\
\hline
\end{tabular}

The morphologic criteria of mass lesions including their shape, margin and pattern of enhancement were assessed with more concern on the margin and enhancement characteristics (Table $6 \& 7$ ).

Table 6 Shows MR margin characteristics of focus and mass lesions as well as its $\mathrm{P}$ value in relation to the histopathology

\begin{tabular}{lllll}
\hline Margin & Benign & Malignant & Total P value \\
\hline Well defined & $6(66.6 \%)$ & $4(33.4 \%)$ & 10 & \\
III defined & I (10\%) & $9(90 \%)$ & 10 & $0.00 \mathrm{I}$ \\
Speculated & $0(0 \%)$ & $1 \mathrm{I}(100 \%)$ & $1 \mathrm{I}$ & \\
Total & $7(23.3 \%)$ & $24(76.7 \%)$ & $3 \mathrm{I}$ & \\
\hline
\end{tabular}

Table 7 Shows the $P$ values of MR enhancement characteristics of focus and mass lesions in correlation with their histopathological results

\begin{tabular}{lllll}
\hline Enhancement pattern & Benign & Malignant & Total P value \\
\hline Homogenous & $2(13.3 \%)$ & $\mathrm{I} 3(86.7 \%)$ & $\mathrm{I} 5$ & $0.62 \mathrm{I}$ \\
Heterogeneous & $\mathrm{I}(9.1 \%)$ & $\mathrm{I} 0(90.9 \%)$ & $\mathrm{II}$ & 0.342 \\
Ring & $0(0 \%)$ & $\mathrm{I}(100 \%)$ & $\mathrm{I}$ & $0.4 \mathrm{I}$ \\
Non enhancing septa & $4(100 \%)$ & $0(0 \%)$ & 4 & $0.00 \mathrm{I}$ \\
Total & $7(23.3 \%)$ & $24(76.7 \%)$ & $3 \mathrm{I}$ & \\
\hline
\end{tabular}

The calculated $P$ value, sensitivity and specificity of mass margin in differentiating benign from malignant was $0.001,93.1 \%$ and $74.3 \%$ respectively. 
Non enhancing internal septae were only found in benign lesions (4 lesions) with calculated $\mathrm{P}$ value was 0.001 . Homogenous enhancement were found in 15 lesions, $2(13.3 \%)$ were benign and $13(86.7 \%)$ were malignant. Heterogeneous enhancement were found in 11 lesions, $1(9.1 \%)$ was benign and $10(90.9 \%)$ were malignant.

Ring enhancement was present in 1 lesion which was proved to be malignant. The calculated $\mathrm{P}$ value of homogenous, heterogenous and ring enhancement was $0.621,0.342$ and 0.001 respectively.

The dynamic behavior of each mass lesion with quantitative analysis of its signal intensity [measured by means of computer manipulated region of interest (ROI)] as well as assessment of the shape of the time/signal intensity curve was studied (Table 8).

Table 8 Shows the minimum, maximum, mean, Std Deviation and P value of the signal intensity increase in benign and malignant masses

\begin{tabular}{llllll}
\hline Pathology & Minimum & Maximum & Mean & Std. Deviation & P value \\
Benign & 25 & 130 & 75 & 31.002 & \\
Malignant & 30 & 280 & 83.13 & 40.639 & 0.671 \\
\hline
\end{tabular}

The calculated $\mathrm{P}$ value of the signal intensity increase was 0.671 with overlapin values between benign and malignant lesions.

The progressive (type I) and wash out (type III) curves were observed in 5 and 0 benign lesions compared to 1 and 22 malignant lesions, respectively. Plateau curve (type II) was present in 6 lesions, $2(33.4 \%)$ was benign and $4(66.6 \%)$ were malignant. The calculated $\mathrm{P}$ value of type I, type II and type III curves was 0.001, 0.891 and 0.000 , respectively (Table 9).

Table 9 Shows the $\mathrm{P}$ value of each type of time/signal intensity curve in relation to histopathology

\begin{tabular}{lllll}
\hline Shape of the Curve & Benign & Malignant & Total P value \\
\hline Progressive rising & $5(83.4 \%)$ & $\mathrm{I}(16.6 \%)$ & 6 & $0.00 \mathrm{I}$ \\
Plateau & $2(33.4 \%)$ & $4(66.6 \%)$ & 6 & $0.89 \mathrm{I}$ \\
Wash out & $0(0 \%)$ & $22(100 \%)$ & 22 & 0.000 \\
Total & $7(26.9 \%)$ & $29(73.1 \%)$ & 36 & \\
\hline
\end{tabular}

The overall sensitivity, specificity, PPV, NPV and accuracy of mammography, ultrasonography and dynamic MR mammography (based on BI-RADS system) in differentiating benign from malignant lesions were calculated for all breast lesions (Table 10 \& Figure 2).

Table 10 Compared sensitivity, specificity, PPV, NPV and accuracy of mammography, US and MRM based on BI-RADS category

\begin{tabular}{llll}
\hline & Mammography & US & MRM \\
Sensitivity & $66.7 \%$ & $86.7 \%$ & $98.6 \%$ \\
Specificity & $91.5 \%$ & $87.8 \%$ & $78.8 \%$ \\
PPV & $95.4 \%$ & $94.6 \%$ & $90.6 \%$ \\
NPV & $53.3 \%$ & $66.8 \%$ & $95.3 \%$ \\
Accuracy & $73.1 \%$ & $85.2 \%$ & $91.8 \%$ \\
\hline
\end{tabular}

\section{Illustrative Cases}

Case 1

43 - Year - old diabetic patient with left breast lump.

\section{Mammographic findings}

Craniocaudal and mediolateral oblique views of both breasts showed left breast upper outer soft tissue mass with partially ill defined borders (BIRADS 4a) (Figure 3).
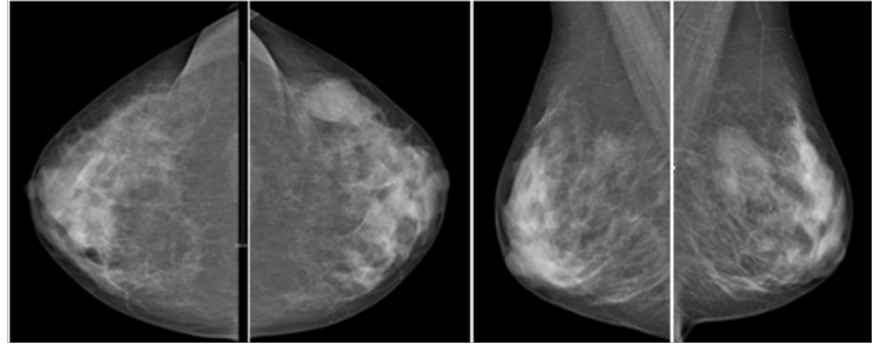

Figure 3 Craniocaudal and mediolateral oblique views of both breasts.

\section{Ultrasonographic findings}

Ultrasound showed left upper outer well defined hypoechoic mass lesion with mild posterior acoustic shadowing (BIRADS 4b) (Figure 4).

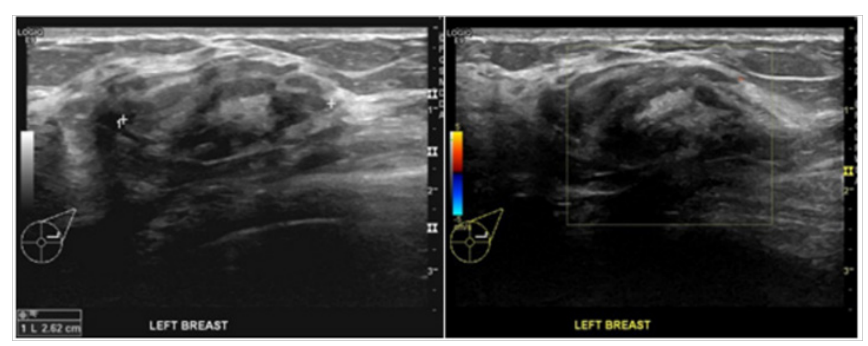

Figure 4 Ultrasound of the left upper outer lesion.

\section{Dynamic MRI findings}

I. T2 FAT SAT images showed left upper outer ill defined area of asymmetry (Figure 5).

II. T1 weighted images showed left upper outer well defined lesion of almost similar or slightly lower signal intensity than the glandular breast tissue (Figure 6).

III. Subtracted dynamic MR images showed left upper outer well defined lesion with homogenous enhancement (Figure 7).

IV. Time /signal intensity curve of the left breast lesion showed type I curve (Figure 8).

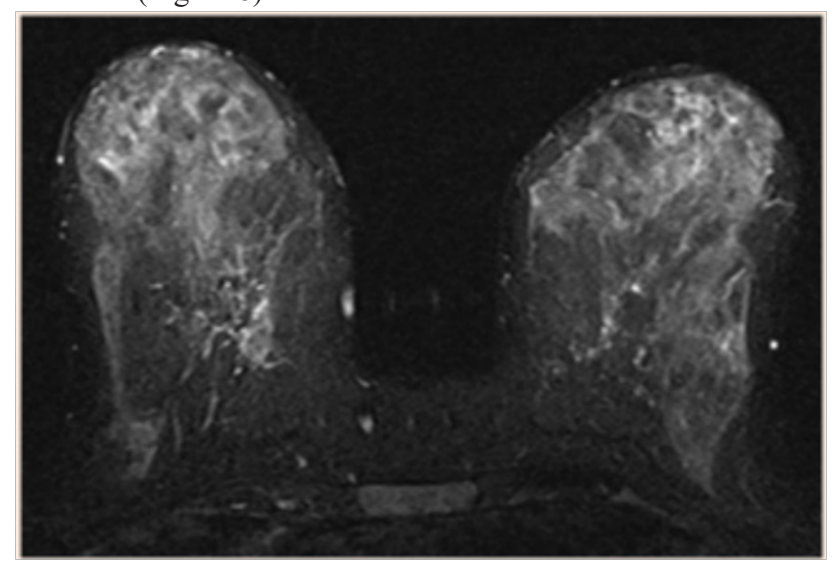

Figure 5 T2 FAT SAT image.

MR BIRADS (Based on morphological appearance and kinetic analysis)

Left breast lesion BIRADS 3. 


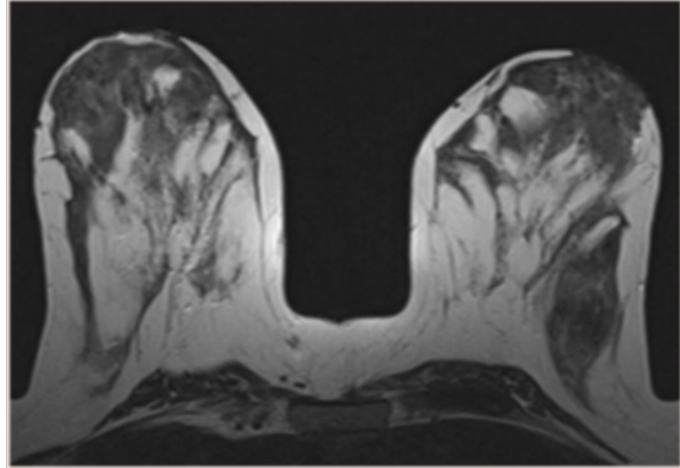

Figure 6 TI weighted image.

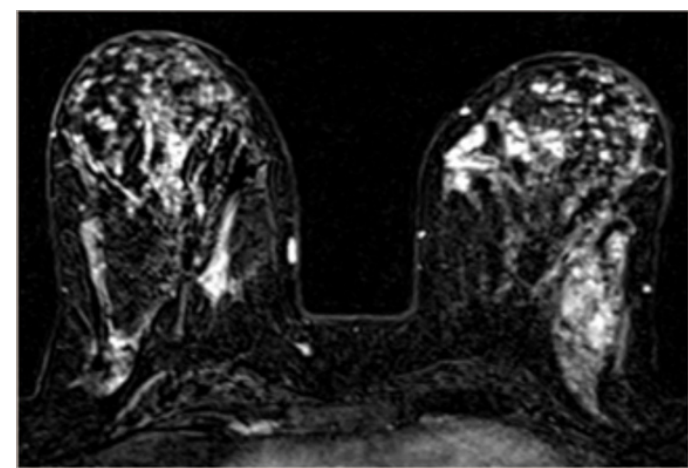

Figure 7 Subtracted dynamic MR image.

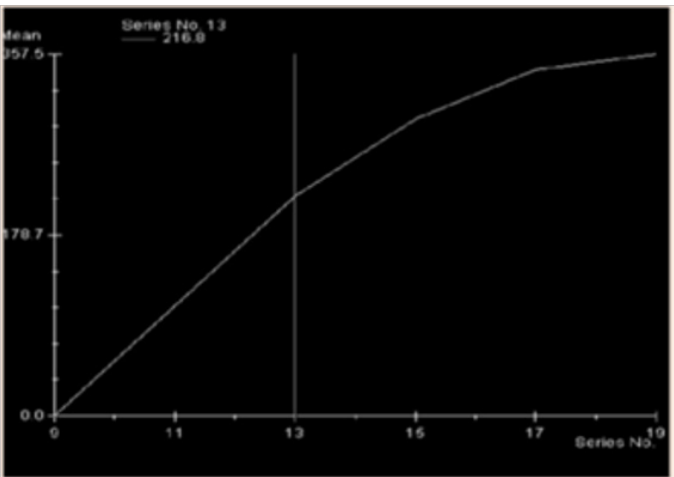

Figure 8 Time /signal intensity curve of the left breast lesion.

\section{Final diagnosis}

The left breast lesion was categorized BIRADS 4a by mammography, BIRADS $4 \mathrm{~b}$ by ultrasonography and BIRADS 3 by MRI. The patient underwent TRU CUT biopsy and histopathology was diabetic mastopathy.

Case 2

48 - year - old patient with left breast lump.

\section{Mammographic findings}

Craniocaudal and mediolateral oblique views of both breasts showed left breast upper outer asymmetrical density (BIRADS 4a) (Figure 9).

\section{Ultrasonographic findings}

Ultrasound showed left upper outer hypoechoic ill define mass lesion with irregular margins and posterior acoustic shadowing (BIRADS 4c) (Figure 10).

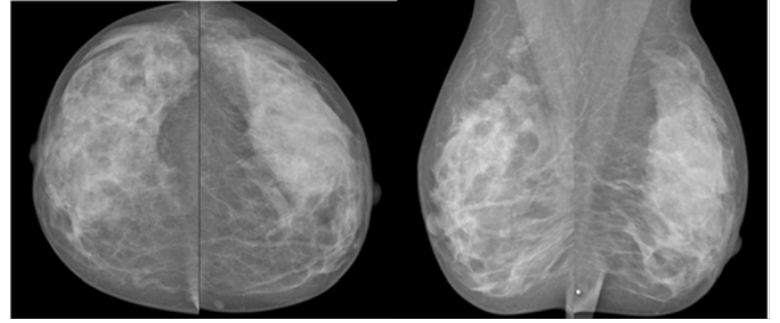

Figure 9 Craniocaudal and mediolateral oblique views of both breasts.
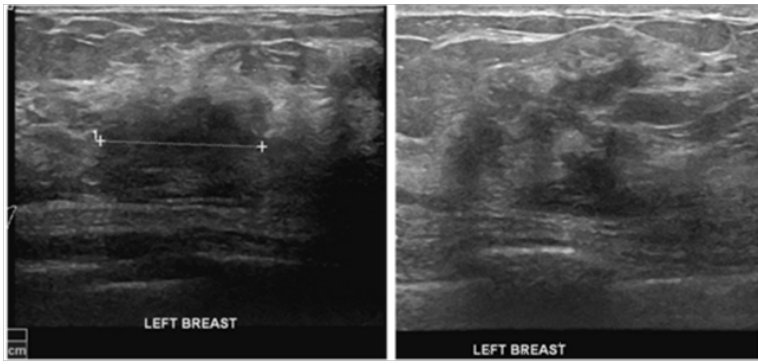

Figure 10 Ultrasound of the left upper outer mass lesion.

\section{Dynamic MRI findings}

I. T2 FAT SAT images showed left breast ill defined area of increased signal intensity (Figure 11).

II. T1WI images showed left breast ill defined area of hypo intensity (Figure 12).

III. Subtracted dynamic MR images showed left upper outer non mass like enhancement (Figure 13).

IV. Time /signal intensity curve of the left breast lesion showed type III curve (Figure 14)

V. MR BIRADS (Based on morphological appearance and kinetic analysis)

VI. BIRADS 5.

VII. Final diagnosis

VIII. The right breast lesion was categorized BIRADS 4a by mammography, BIRADS $4 \mathrm{c}$ by ultrasonography and BIRADS 5 by MRI. The patient underwent TRU CUT biopsy and histopathology was invasive ductal carcinoma.

IX. Case 3

X. 38 - year - old patient with left breast lump.

XI. Mammographic findings

XII. Craniocaudal and mediolateral oblique views of both breasts showed left breast upper outer ill defined area of asymmetrical density (BIRADS 4c) (Figure 15).

XIII. Ultrasonographic findings

XIV. Ultrasound showed left upper outer ill definedhypoechoic lesion with irregular margins and posterior acoustic shadowing (BIRADS 4c) (Figure 16).

\section{Dynamic MRI findings}

I. T2 FAT SAT images showed left upper outer hyperintense mass lesion with irregular margins (Figure 17). 
II. Pre contrast T1 FAT SAT FLASH images showed left upper outer mass lesion with irregular margins (Figure 18).

III. Subtracted dynamic MR images showed left upper outer mass lesion with homogenous enhancement and irregular margins (Figure 19).

IV. Time /signal intensity curve of the left breast lesion showed type II curve (Figure 20).

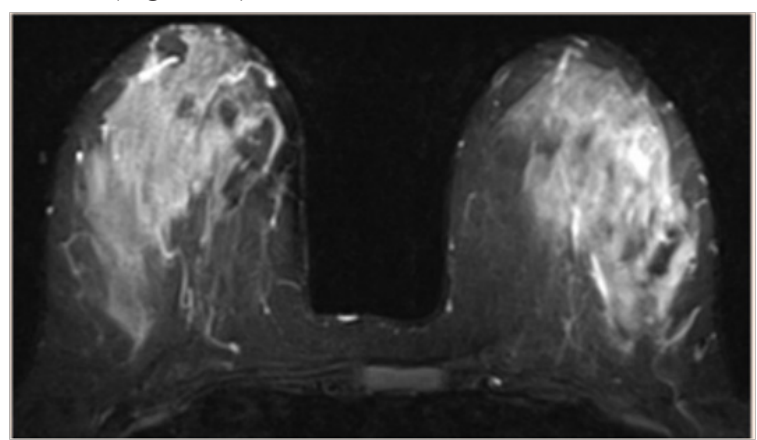

Figure I I T2 FAT SAT image.

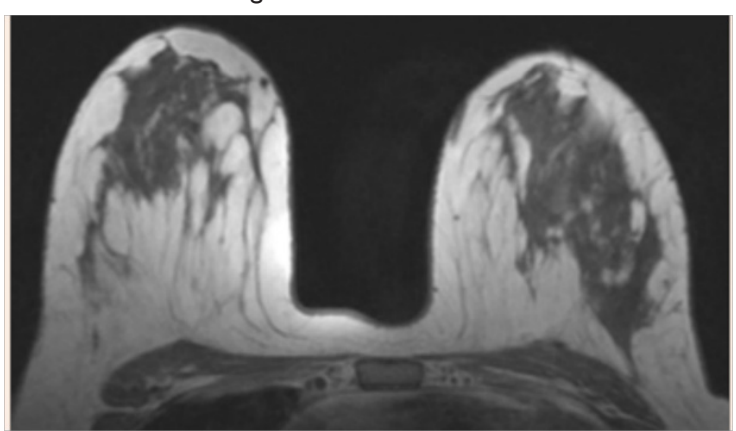

Figure $12 \mathrm{TIWI}$ image.

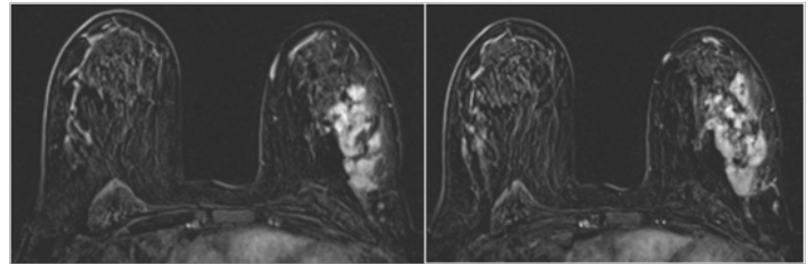

Figure 13 Subtracted dynamic MR images.

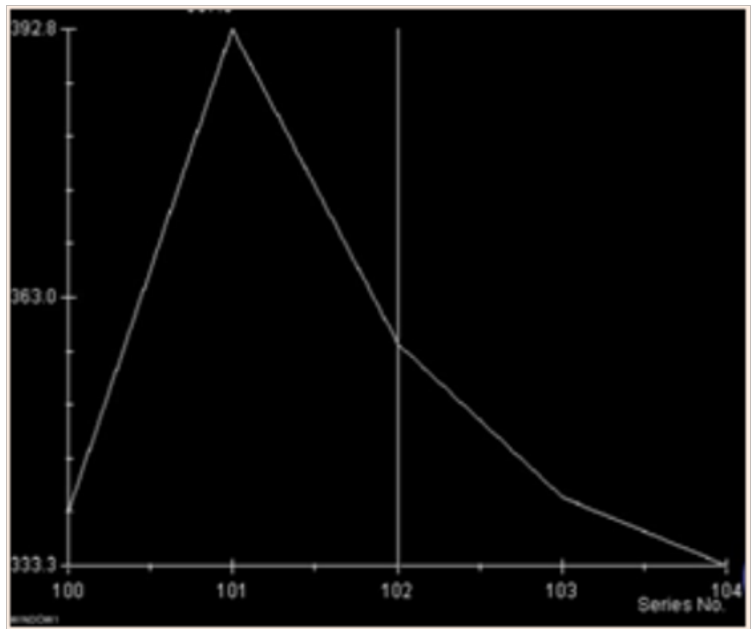

Figure 14 Time/signal intensity curve of the left breast lesion.

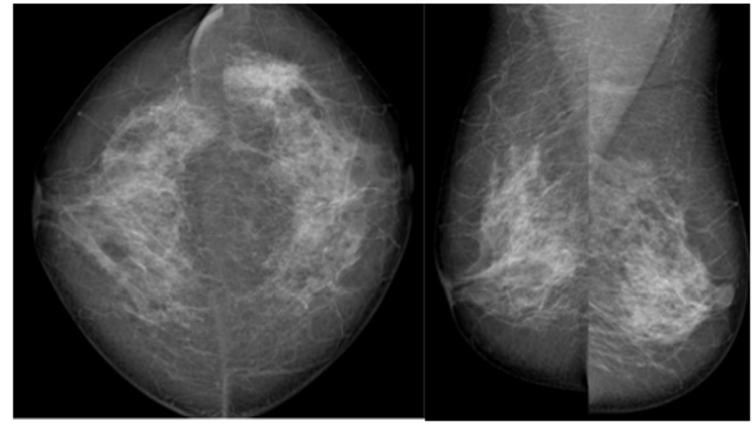

Figure 15 Craniocaudal and mediolateral oblique views of both breasts.

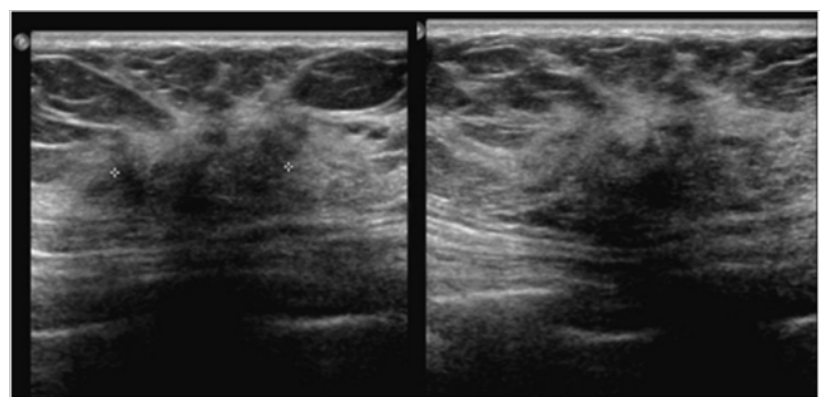

Figure 16 Ultrasound of the left breast lesion.

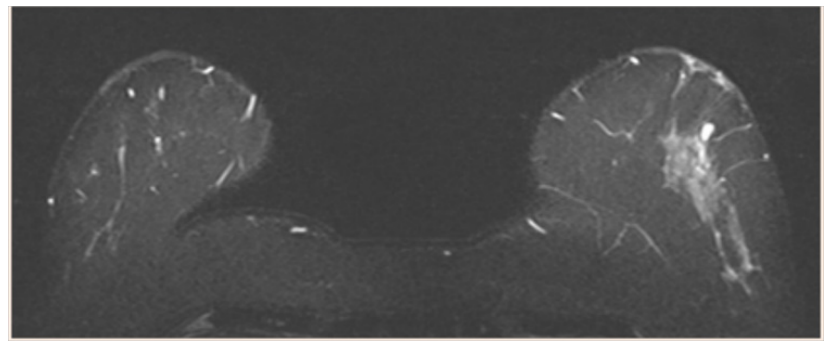

Figure 17 T2 FAT SAT image.

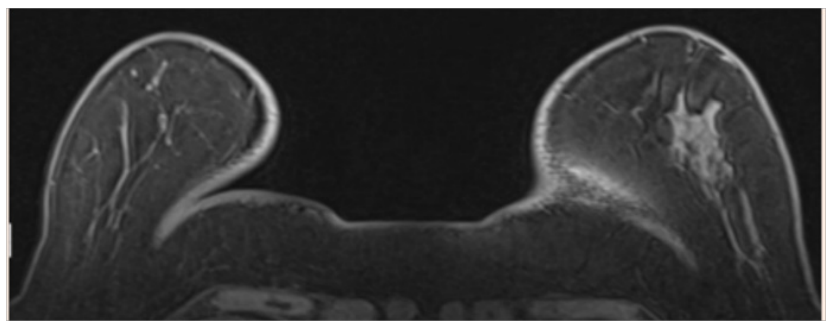

Figure I 8 Pre contrast TI FAT SAT FLASH image.

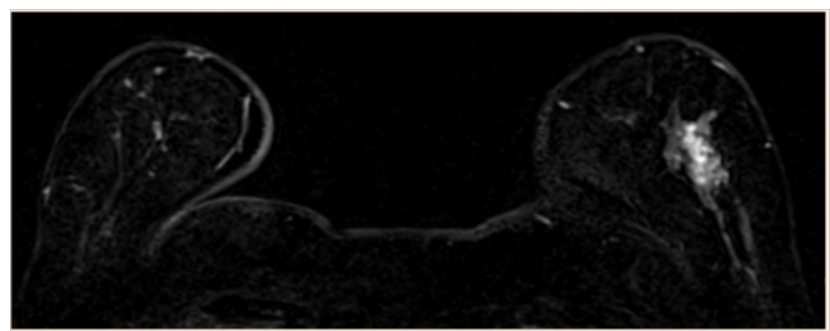

Figure 19 Subtracted dynamic MR image.

\section{MR BIRADS (Based on morphological appearance and} kinetic analysis)

Left breast lesion BIRADS 5. 


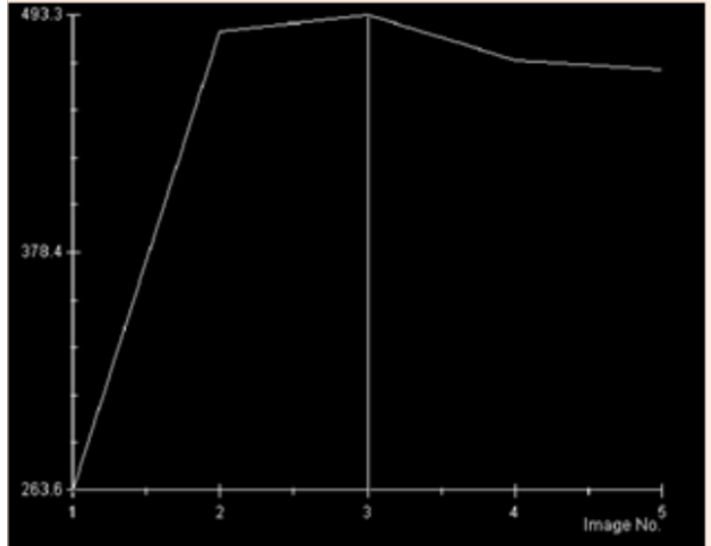

Figure 20 Time /signal intensity curve of the left breast lesion.

\section{Final diagnosis}

The left breast lesion was categorized BIRADS $4 \mathrm{c}$ by mammography, BIRADS $4 \mathrm{c}$ by ultrasonography and BIRADS 5 by MRI. The patient underwent TRU CUT biopsy and histopathology was invasive ductal carcinoma.

\section{Case 4}

44 - Year - old patient with left breast palpable mass.

\section{Mammographic findings}

Craniocaudal and mediolateral oblique views of both breasts (Figure 21) as well as magnification view (Figure 22) showed left deep retroareolar well defined dense lesion (BIRADS 3).

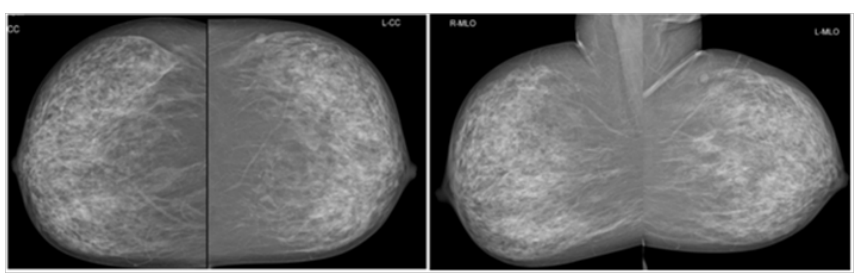

Figure 2I Craniocaudal and mediolateral oblique views of both breasts.

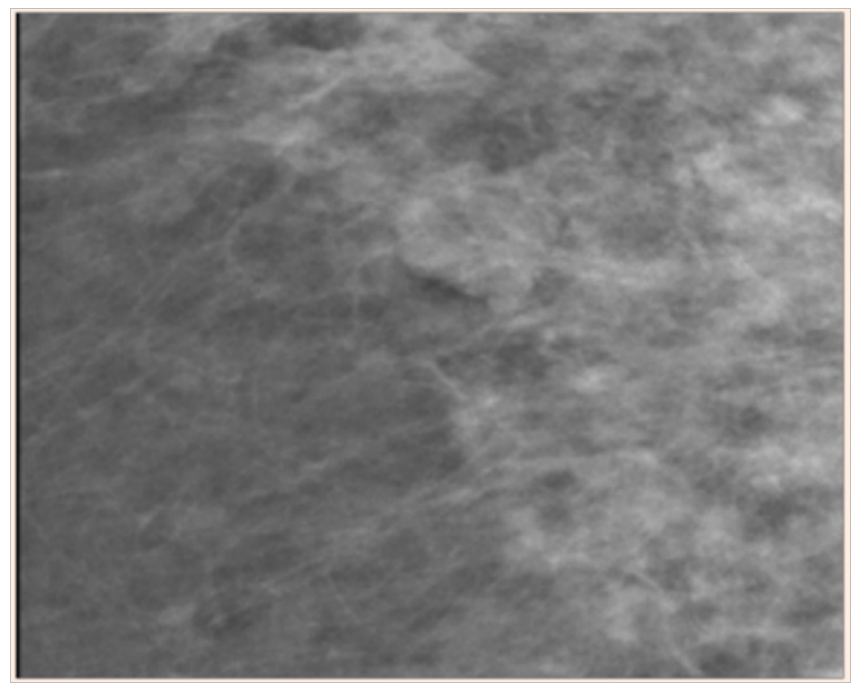

Figure 22 Magnification view of the left breast deep retroareolarlesion.

\section{Ultrasonographic findings}

Ultrasound images showed left breast hypoechoic lesion with irregular margins (BIRADS 4b) (Figure 23).
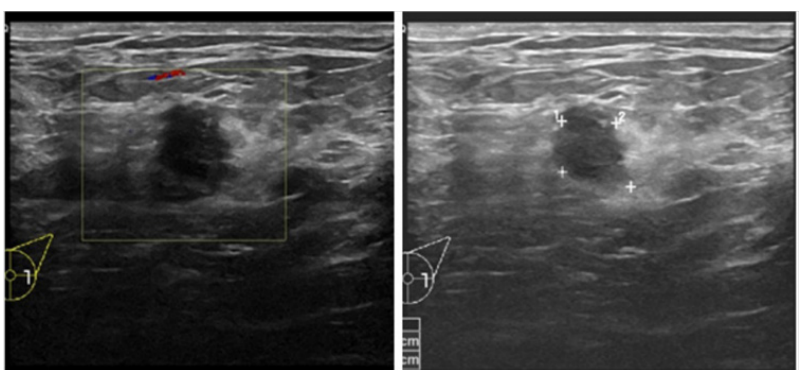

Figure $\mathbf{2 3}$ Ultrasound images of the left breast lesion.

\section{Dynamic MRM findings}

I. T2 FAT SAT images showed left breast deep retro-areolar well defined hyperintense lesion (Figure 24).

II. The lesion appeared hypointense in T1WI (Figure 25).

III. Subtracted dynamic MR images showed homogenous enhancement of the lesion (Figure 26).

IV. Time /signal intensity curve of the left breast lesion showed type III curve (Figure 27).

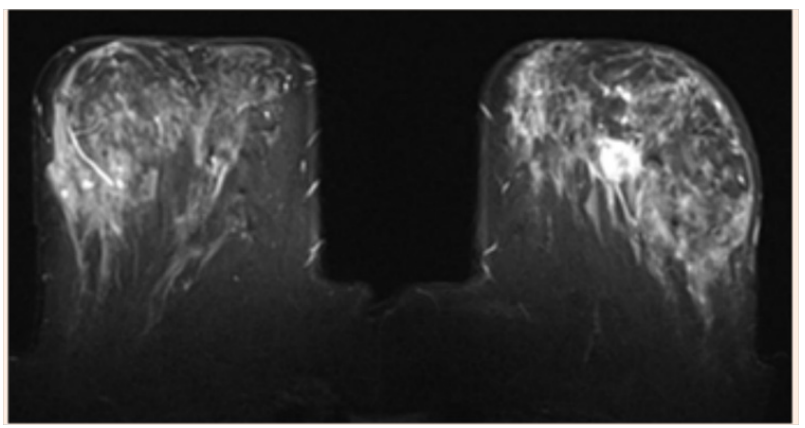

Figure 24 T2 FAT SAT image.

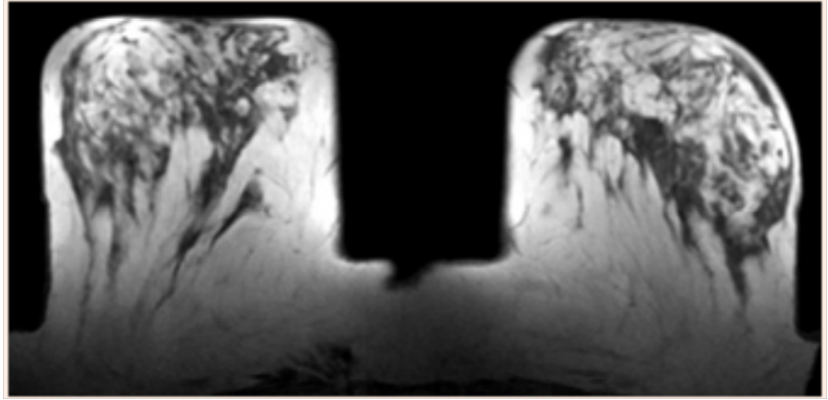

Figure $\mathbf{2 5}$ The lesion in TIWI.

MR BIRADS (Based on morphological appearance and kinetic analysis)

BIRADS 4.

\section{Final diagnosis}

The left breast lesion was categorized BIRADS 3 by mammography, BIRADS $4 b$ by ultrasonography and BIRADS 4 by MRI. The patient underwent TRU CUT biopsy and histopathology was medullary carcinoma. 


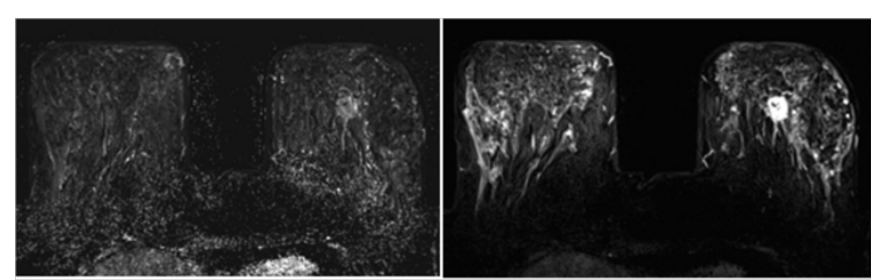

Figure 26 Subtracted dynamic MR images.

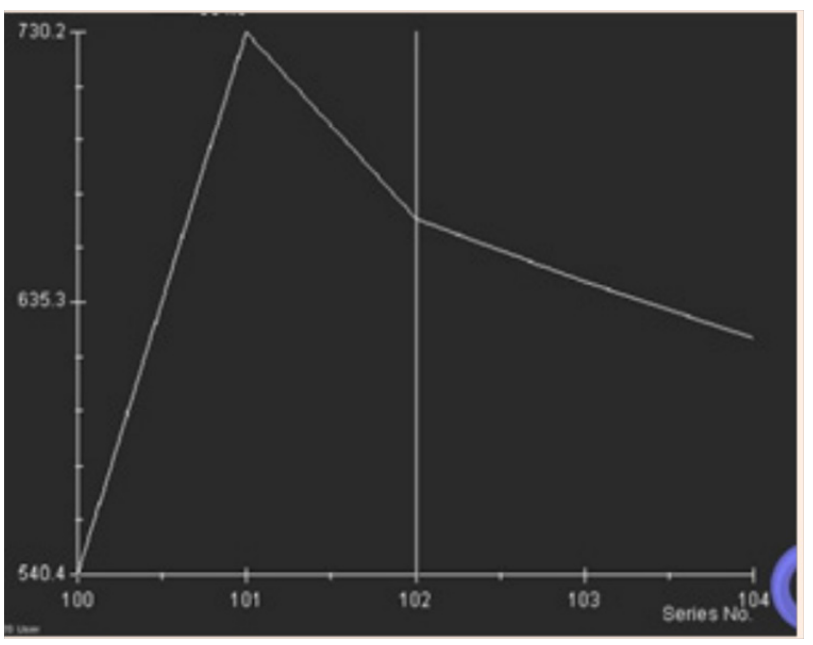

Figure 27 Time /signal intensity curve of the left breast lesion.

\section{Discussion}

Breast cancer is a leading oncologic cause of death in women. ${ }^{9}$ It is generally accepted that early detection of breast cancer increases the probability of cure. ${ }^{10}$ The objective of traditional diagnostic imaging examinations is to estimate the extent of disease and identify subclinical disease in the affected and contralateral breast to guide local therapy. ${ }^{11}$

Mammography has been shown to reduce breast cancer mortality in population-based screening programs however, it has well-recognized limitations, such as exposure to ionizing radiation and low sensitivity in patients with dense breasts. ${ }^{12}$

Ultrasonography has undergone significant improvements in the last years that have extended its utility for breast imaging. Important clinical advances in breast US have been the improved benign/ malignant differentiation of solid breast lesions and the use of US to guide interventional procedures such as needle aspirations, coreneedle biopsies, and pre-biopsy needle localizations of breast masses or calcifications. Extended field of view imaging provides panoramic high-resolution images of the entire breast. Tissue harmonic imaging has the potential to improve lesion-background contrast and proximal resolution both for breast lesions and in particular the axilla, resulting in an improvement in overall image quality despite some problems with posterior acoustic shadowing. ${ }^{13}$

The role of MRI in evaluating breast disease has been studied and debated since contrast enhanced MRI was introduced in 1985. Interest has grown steadily as evidence of its usefulness has accumulated. ${ }^{14}$

Contrast enhanced magnetic resonance imaging (CE-MRI) with its high soft tissue contrast, multiplanar sectioning and three dimensional representation of the breast provides high sensitivity (over 90\%) in the detection of breast cancer. However, the specificity for lesion characterization is still low to moderate (72\%), turning the discrimination between cancer and benign lesions into a challenge. The main additional diagnostic value of MRI relies on (1) detecting foci of multifocal, multicentric or contralateral disease unrecognized on conventional assessment (physical examination, mammography and ultrasound); (2) recognition of invasive components in ductal carcinoma in situ (DCIS); (3) assessing the response to neoadjuvant chemotherapy (NAC); (4) detecting an occult primary breast cancer in patients presenting with metastatic cancer in axillary nodes; and (5) detection of cancer in dense breast tissue. ${ }^{15}$

We conducted a study of 33 patients with thirty six breast lesions to evaluate the role of DCE MRI in the probably benign and suspicious breast findingsafter mammographic and ultrasonographic examinations. Mammographic evaluation of the lesions was based on Breast Imaging Reporting and Data System (BI-RADS) with classification of these lesions into BI-RADS 0which was assigned for six lesions with very dense breast. BI-RADS 3 and BI-RADS 4 were our main concern in this study and were encountered in six and seventeen lesions respectively.

In our study, mammographic examination of the breast lesions yielded an overall sensitivity of $66.7 \%$, a specificity of $91.5 \%$ and accuracy of $73.1 \%$. Our results are comparable to previous studies reported the sensitivity of mammography to be $71.8 \%$ and thespecificity to be $97 \% .^{16}$

We agreed with Le-Petross et al. ${ }^{17}$ that the breast density is one of the most important factors limiting the sensitivity of mammography and that was encountered in six cases in our study. In our study the high specificity of mammography may be attributed to selection of cases unlike studies which evaluated general population and healthy women who undergo periodic screening. Mammography is extremely sensitive in detecting microcalcifications even though it is inadequate for differentiating malignant from benign lesions and invasive from in situ carcinomas. ${ }^{18}$

In our study we encountered five lesions with micro calcifications, four of them were given mammographic BIRADS score of 4 and one was given a BIRADS score of 5. The histopathological results yielded 3 cases of invasive ductal carcinoma and two cases of DCIS.

In our study all patients were examined by ultrasound with classification of their breast lesions according to ultrasound BI-RADS lexicon. The overall calculated ultrasound sensitivity, specificity and accuracy were $86.7 \%, 87.8 \%$ and $85.2 \%$ respectively.

In a study done by Vassou et al. ${ }^{19}$ reported that ultrasound sensitivity and specificity were $83 \%$ and $76 \%$, respectively. Several prospective studies have reported increased tumor detection in high-risk women who undergo MRI as part of their breast cancer screening program. ${ }^{11}$

MRI has emerged as an alternative, powerful tool for breast cancer screening as it does not require exposure to ionizing radiation; it is thus safe to use routinely and more suitable than mammography for assessing young women. 11Breast MRI is a clinically useful additional diagnostic tool and has an excellent sensitivity and negative predictive value (NPV), which usually exceeds $90 \%$. However, the overall specificity of breast MRI varies between $67 \%$ and $72 \%$. The diagnostic accuracy of breast MRI varies with the expertise of the radiologist and the particular patient population studied. ${ }^{20}$

Different investigators described that certain dynamic signal intensity (SI) characteristics (rapid and intense contrast enhancement followed by a washout phase) obtained in dynamic studies are a 
strong indicator for malignancy. Morphological criteria have also been identified as valuable diagnostic tools. Recently, combinations of different dynamic and morphological characteristics have been reported that can reach diagnostic sensitivities up to $97 \%$ and specificities up to $76.5 \% .^{21}$

In our study we combined both morphologic and dynamic parameters and its modification into BI-RADS category for lesion classification. The sensitivity of MRI examinations was $98.6 \%$ while the specificity was $78.8 \%$, this was attributed to the small number of the benign lesions, representing 7(19.4\%) out of 36 examined lesions.

In general, margin and shape analysis should be performed on first post contrast image to avoid wash out and progressive enhancement of the surrounding breast tissue. ${ }^{22}$ The description of the margin of a focal mass is the most predictive feature of the breast MR image interpretation. Speculated margins are suspicious for carcinoma, having $91 \%$ positive predictive value (PPV) for malignancy. ${ }^{23}$

In our study speculated margins having (100\% specificity and $100 \%$ PPV) being encountered only in malignant lesions. The calculated $\mathrm{P}$ value, sensitivity and specificity of mass margin in differentiating benign from malignant was $0.001,93.1 \%$ and $74.3 \%$ respectively. Some of the most powerful diagnostic criteria for the differentiation of benign and malignant tumors belong to internal enhancement of focal mass. ${ }^{24}$

The evaluation of the enhancement from the quantitative and qualitative points of view is in fact the assessment of vascularization of the lesion. The attribute of neoangiogenesis is used in malignant lesions which are often too small to be proved by another imaging method. ${ }^{25}$

In our study non enhancing internal septations were only found in benign lesions (4 lesions) proved to be fibroadenomas by histopathology. The calculated $\mathrm{P}$ value of non enhancing internal septations was statistically significant $(<0.005)$. Kuhl ${ }^{24}$ reported that dark septation if present within a lobular or oval mass are typical of fibroadenomas.

Tozaki et al. ${ }^{26}$ reported that the most frequent morphological finding among the malignant lesions was heterogeneous internal enhancement. In our study one benign lesion exhibited heterogeneous enhancement, its pathological diagnosis was diabetic mastopathy. Ring enhancement was found in one malignant case. Homogenous enhancement was found in 15 lesions, $2(13.3 \%)$ were benign and $13(86.7 \%)$ were malignant. In our study there was no statistical correlation between the pathologically proven benign and malignant lesions regarding their enhancement pattern except the non enhancing internal septations and this may be attributed to the small number of the studied patients, however our all pathologically proven benign cases did not show enhancing internal septations (100\% specificity).

Imamura et al. ${ }^{27}$ found in his study that malignant non mass lesions tended to show either segmental or branching ductal distribution, and when lesions with these patterns are considered malignant the sensitivity \& specificity were $68.8 \%$ and $63.6 \%$ respectively. He also reported that using the enhancement pattern in differentiation between benign and malignant lesions is often difficult with non mass like enhancement as there is no standarized method for interpreting them.

We encountered five lesions of non mass like enhancement, all of them proved to be malignant; two lesions were of linear ductal enhancement and proved pathologically to be invasive ductal carcinoma. The remaining three lesions showed segmental stippled enhancement, two of them proved to be ductal carcinoma in situ and the other one proved to be invasive ductal carcinoma.

When planning a surgical strategy for treating preoperatively diagnosed cases of DCIS, it is clinically important to be able to more accurately predict the presence or absence of unexpected invasive disease in surgical specimens. ${ }^{28}$

The most common presentations of DCIS are the malignant micro calcifications in mammography. ${ }^{23}$ However, mammographic estimates, based on the extent of micro calcifications, frequently under estimates tumour size. Additionally, mammography does not reliably demonstrate the extent of un calcified DCIS. ${ }^{29}$

In the case of DCIS manifesting by micro calcifications in mammography, the real advantage of breast MRI is the ability to evaluate accurately the micro calcification lesion and even the mammographic ally occult area without micro calcifications by detecting the DCIS with micro invasion. ${ }^{25}$

Very typical morphological feature for high but also for low DCIS is ductal pattern of enhancement. Usually it is clumped, and the heterogeneous or homogeneous enhancement sometimes can be of branching appearance and follows the course of the duct. The enhancement can also have a non regular stippled, spotty and micro nodular appearance. Segmental and linear character of this enhancement is described and this morphological finding was assessed to have high PPV for DCIS lesions. ${ }^{25}$

In our study we encountered on mammography five lesions with micro calcifications; three of them were histopathologically invasive ductal carcinoma and two lesions were DCIS. On DCE MRI, The two cases of DCIS had segmental stippled non mass like enhancement and the other three cases were associated with masses exhibiting heterogenous enhancement. The MRI sensitivity for microcalcifications detection was $85.6 \%$ while specificity was $87.3 \%$.

Yet our study population is small to yield accepted specificity results recommending further research in determining the role of MRI in suspicious micro calcifications. It was assessed that MRI morphologic features of DCIS can reflect differences in biology and pathology of these tumours. The region of interest (ROI) which is necessary to gain relevant quantitative and qualitative evaluation of enhancement must be very small in this ductal pattern of enhancement to obtain the most accurate measurement. The ROI must cover only part of the lesion, and should be placed in the region of strongest enhancement on the first contrast-enhanced image. When the ROI is randomly placed in the mass, the enhancement curve may be variable and yield lower specificity however, regarding the detection of ductal carcinoma in situ (DCIS), the sensitivity of MRI varies between $70 \%$ and $100 \%$ according to the latest studies. ${ }^{25}$

It was shown that, MRI had relatively high sensitivity for high grade DCIS which often showed rapid initial rise and enhancement pattern that resembles invasive carcinoma by its characteristics. Low grade DCIS had often quite non typical form of enhancement pattern and initial rise was usually lower and is of course difficult to differentiate it from benign proliferative processes that often enhance on MRI. It has been pointed out that $10 \%$ of the DCIS did not enhance at all. Moreover, $30 \%$ of the enhancing DCIS showed a nonspecific enhancement that cannot be distinguished from fibrocystic breast disease..$^{25}$

Our study encountered four cases of DCIS two of them had segmental stippled non mass like enhancement and the other two 
showed small masses with homogenous enhancement and speculated outline with a sensitivity of $77.3 \%$.

In our study three of the four cases of DCIS showed rapid initial peak of enhancement followed by wash out pattern (Type III curve) similar to the contrast enhancement behavior of invasive carcinoma while one case showed delayed initial peak of enhancement followed by plateau (Type II curve).

If the ductal pattern of enhancement (during negative mammography and ultrasound finding) and kinetic curve assessment suspicious of malignancy are found in MRI, most of the authors recommend biopsy under MRI control. Some authors recommend further work-up in case only if the segmental or ductal pattern of enhancement is identified on breast MRI. They recommend either direct MR-guided vaccum-assisted biopsy or short-term follow-up breast MRI within three months. ${ }^{25}$

In our study there was an overlap in the enhancement rate of benign and malignant lesions ranged from 25 to $120 \%$ (in benign lesions) and from 30 to $280 \%$ (in malignant lesions). Our calculated $\mathrm{P}$ value was insignificant $(>0.005)$, this is comparable with the study of Kuhl et al. ${ }^{24}$ who reported that enhancement rates proved to be not diagnostically relevant because the broad overlap between benign and malignant lesions and were therefore of only limited diagnostic use in the individual patient.

The curve shape is an important differentiator between cancer and benign lesions for comparable enhancement rates. The majority of benign lesions have a Type I curve, and the majority of malignant lesions have a Type III curve. Unfortunately there is overlap within these categories. ${ }^{12}$

We calculated the $P$ value of each type of time signal intensity curve and we found that progressive (type I ) and the wash out (type III) curves were found in $5(83.4 \%)$ and $0(0 \%)$ pathologically proven benign lesions compared to $1(16.6 \%)$ and $22(100 \%)$ malignant lesions respectively. So their calculating $\mathrm{P}$ value was significant $(<0.005)$ in differentiation benign from malignant lesions with progressive type curve more observed in benign findings and wash out curve more with malignant findings. In contrary, the P value of plateau (type II) was insignificant $(>0.005)$ being present in $2(33.4 \%)$ benign lesions compared to $4(66.6 \%)$ malignant lesions.

Actually in our interpretation the main concern was given to the morphological assessment rather than the time intensity curve one due to its limited specificity yet it was considered strong positive indicator in case of type 1 curve seen in pathologically proven benign or type 3 curve seen in pathologically proven malignant lesions.

Our results were comparable with the study of Seely et al. ${ }^{30}$ reported that BI-RADS category in breast MRI had the highest combination of specificity and sensitivity $(77.1 \%$ and $81.8 \%)$ as well as the highest interobserver correlation.

Leung J [31]stated that MR imaging has high negative predictive value in excluding breast cancer, so it plays a role in the evaluation of selected clinical and imaging findings of the breast, especially when biopsy is not technically feasible. Case selection is very important in ensuring the efficacy of this use of MR imaging because of potential false-positive and false-negative results.

In our study the overall sensitivity of mammography, US and MRI was $66.7 \%, 86.7 \%$ and $98.6 \%$ respectively. Their specificity was $91.5 \%, 87.8 \%$ and $78.8 \%$ respectively. MRI was the most sensitive imaging method for detection of cancer but with limited specificity due to overlap in features of benign and malignant lesions. Ultrasound was the imaging modality with intermediate sensitivity and specificity.

\section{Conclusion}

The purpose of all diagnostic modalities in breast cancer is early detection and proper diagnosis, which has its major impact on further management and prognosis. The current study evaluated the role of MRM in suspicious breast lesions diagnosed by mammography and ultrasound examination. In our study MRI was the most sensitive imaging method for detection of cancer but with limited specificity due to overlap in features of benign and malignant lesions.

\section{Conflicts of Interest}

The authors declare no conflict of interest.

\section{Acknowledgments}

None.

\section{Funding}

None.

\section{References}

1. Imbriaco M, Caprio MG, Limite G, et al. Dual-Time-Point 18F-FDG PET/ CT Versus Dynamic Breast MRI of Suspicious Breast Lesions. AJR. 2008;191(5):1323-1330.

2. Shah S, Shah S, Greatrex K. Current Role of Magnetic Resonance Imaging in Breast Imaging: A Primer for the Primary Care Physician. $J$ Am Board Fam Pract. 2005;18(6):478-490.

3. Peng KQ, Huang ZL, Xie CM, et al. Application of dynamic contrast enhancement MRI and post-processing technique for diagnosis of breast cancer. Chinese J of Cancer. 2009;28(5):549-554.

4. Guinea OF, Andicoechea A, González LO, et al. Relationship between morphological features and kinetic patterns of enhancement of the dynamic breast magnetic resonance imaging and clinico-pathological and biological factors in invasive breast cancer. BMC Cancer. 2010;10:8.

5. Lee SH, Cho N, Kim SJ, et al. Correlation between High Resolution Dynamic MR Features and Prognostic Factors in Breast Cancer. Korean J Radiol. 2008;9(1):10-18.

6. Türkbey B, Thomasson D, Pang Y, et al. The role of dynamic contrastenhanced MRI in cancer diagnosis and treatment. Diagn Interv Radiol. 2010;16(3):186-192.

7. Houserkova D, Prasad SN, Svach I, et al. The value of dynamic contrast enhanced breast MRI in mammographically detected BI-RADS 5 microcalcifications. Biomed Pap Med Fac Univ Palacky Olomouc. 2008;152(1):107-115.

8. DeMartini W, Lehman C, Partridge S. Breast MRI for Cancer Detection and Characterization: A Review of Evidence-Based Clinical Applications. Acad Radiol. 2008;15(4):408-416.

9. Olsen L, Morton M, Stan D, et al. Is There a Role for Magnetic Resonance Imaging in Diagnosing Palpable Breast Masses When Mammogram and Ultrasound Are Negative? Journal of women's health. 2012;21(11):1149-1154.

10. Drukteinis JS, Mooney BP, Flowers CI, et al. Beyond Mammography: New Frontiers in Breast Cancer Screening. Am J Med. 2013;126(6):472479 .

11. Breslin T, Banerjee M, Gust C, et al. Trends in Advanced Imaging Use for Women Undergoing Breast Cancer Surgery. Cancer. 2013;119(6):12511256 . 
12. Heller S, Moy L, Lavianlivi S, et al. Differentiation of Malignant and Benign Breast Lesions Using Magnetization Transfer Imaging and Dynamic Contrastenhanced MRI. J Magn Reson Imaging. 2013;37(1):138-145.

13. Andreea G, Pegza R, Lascu L, et al. The Role of Imaging Techniques in Diagnosis of Breast Cancer. Current health sciences journal. 2011;37(2):55-61.

14. Enriquez L, Listinsky J. Role of MRI in breast cancer management Cleve Clin J Med. 2009;76(9):525-532.

15. Menezes GL, Bosch VD, Postma EL, et al. Invasive ductolobular carcinoma of the breast:spectrum of mammographic, ultrasound and magnetic resonance imaging findings correlated with proportion of the lobular component. Springer Plus. 2013;2:621-632.

16. Elsamaloty H, Elzawawi MS, Mohammad S, et al. Increasing accuracy of detection of breast cancer with 3 T MRI. Am J Roentgenol. 2009;192(4):1142-1148.

17. Le-Petross HT, Whitman GJ, Atchley DP, et al. Effectiveness of Alternating Mammography and Magnetic Resonance Imaging for Screening Women With Deleterious BRCA Mutations at High Risk of Breast Cancer. Cancer. 2011;117(17):3900-3907.

18. Fiaschetti V, Pistolese CA, Perretta T, et al. 3-5 BI-RADs Microcalcifications: Correlation between MRI and Histological Findings. ISRN Oncol. 2011:643890.

19. Vassiou K, Kanavou T, Vlychou M, et al. Characterization of breast lesions with CE-MR multimodal morphological and kinetic analysis: Comparison with conventional mammography and high resolution US. European J of Radiology. 2009;70:69-76.

20. Dorrius MD, Pijnappel RM, et al. Breast magnetic resonance imaging as a problem-solving modality in mammographic BI-RADS 3 lesions. Cancer Imaging. 2010;10: S54-S58.

21. Hoffmann S, Shutler J, Lobbes M, et al. Automated analysis of nonmass-enhancing lesions in breast MRI based on morphological, kinetic, and spatio-temporal moments and joint segmentation-motion compensation technique. EURASIP Journal on Advances in Signal Processing. 2013;2013:172.

22. Morris E. Breast MR imaging lexicon updated. Magn Reson Imaging Clin N Am. 2006;14(3):293-303

23. Macura K, Ouwerkerk R, Jacobs M,et al. Patterns of Enhancement on Breast MR Images: Interpretation and Imaging Pitfalls. RadioGraphics. 2006;26(6):1719-1734.

24. Kuhl C. Concepts for differential diagnosis in breast MR imaging. Magn Reson Imaging Clin N Am. 2006;14(3):305-328.

25. Turnbull L, Brown S, Harvey I, et al. Comparative effectiveness of MRI in breast cancer (COMICE) trial: a randomised controlled trial. Lancet. 2010;375(9714):563-571.

26. Tozaki M, Igarashi T, Fukuda K Positive and negative predictive values of BI-RADS descriptors for focal breast masses. Magn Reson Med Sci. 2006;5(1):7-15.

27. Imamura T, Isomoto I, Sueyoshi E, Yano H, Uga T, et al. Diagnostic performance of ADC for non mass like breast lesions on MR imaging. Mag Reso. Med Sci. 2010;9(4):217-225.

28. Miyashita M, Amano G, Ishida T, et al. The Clinical Significance of Breast MRI in the Management of Ductal Carcinoma In Situ Diagnosed on Needle Biopsy. Jpn J Clin Oncol. 2013;43(6):654-663.

29. Barreau B, Mascarel ID, Feuga C, et al. Mammography of ductal carcinoma in situ of the breast: review of 909 cases with radiographicpathologic correlations. Eur J Radiol. 2005;54(1):55-61.

30. Seely JM, Nguyen ET, Jaffey J. Breast MRI in the evaluation of locally recurrent or new breast cancer in the postoperative patient: Correlation of morphology and enhancement features with the BI-RADS category. Acta Radiol. 2007;48(8):838-845.

31. Leung J. MR Imaging in the evaluation of equivocal clinical and imaging findings of the breast. MRI Clin N Am. 2010;18(2): 295-308. 